\title{
THE ROLE OF PAHS IN THE PHYSICS OF THE INTERSTELLAR MEDIUM
}

\author{
L. Verstraete ${ }^{1}$
}

\begin{abstract}
In the interstellar medium (ISM), PAHs are abundant and also carry most of the dust surface. They are thus privileged sites for surface reactions such as the formation of $\mathrm{H}_{2}$. In regions penetrated by UV photons, PAHs loose electrons by the photoelectric effect and efficiently heat the gas. In more shielded regions, PAH recombine and may carry an important fraction of the cloud electronic charge which plays an important role in the gas dynamics and chemistry. We review here processes involving PAHs which control key aspects of the physics of the ISM. We also discuss the corresponding observational constraints. Most of these processes involve a detailed knowledge of the charge of PAHs and we therefore review current models in this area. We argue that more laboratory measurements of the rate of electronic capture on large PAH cations are needed.
\end{abstract}

\section{Introduction}

The interstellar lifecycle brings matter from low-density medium (the diffuse ISM or atomic HI gas heated by the standard radiation field) to dense, molecular clouds and eventually protostars. Thanks to the mechanical action of evolved stars (winds, supernovae), molecular clouds are dispersed and restored to the diffuse ISM. Intimately mixed to the gas, dust and in particular the small grains (of radius $a \leq 10 \mathrm{~nm}$, which include PAHs) play an important role in the thermodynamics, dynamics and chemistry of the ISM. Dust is also the main source of extinction and thus controls the radiative transfer in interstellar clouds.

The primary effect of the interstellar lifecycle on dust is to change the abundance of small grains with respect to larger ones (radii from 10 to a few $100 \mathrm{~nm}$ ) during fragmentation (by radiation or shocks in the diffuse ISM) and growth (by coagulation in dense regions) episodes. In the vicinity of young stars or in shocks PAHs are observed to be depleted (Geers et al. 2006, 2009; Peeters et al. 2005;

1 Institut d'Astrophysique Spatiale, UMR 8617, University Paris-Sud 11, Bât. 121, 91405 Orsay Cedex, France 
Lebouteiller et al. 2007; see Acke et al.; Rho et al., Sandstrom et al. and Siebenmorgen this volume). PAH coagulation is suggested by observations of molecular clouds (Boulanger et al. 1994; Flagey et al. 2009). Conversely, PAHs may be photoevaporated off larger grains when fresh molecular material is exposed to stellar UV radiation thus producing a limb brightening effect (Boulanger et al. 1990; Bernard et al. 1993; Rapacioli et al. 2005; Berné et al. 2007; Compiègne et al. 2008; Velusamy \& Langer 2008; Rapacioli et al. this volume).

In the following, we first review interstellar processes where PAHs play an important role and discuss the observational evidence. The next section is devoted to a key property of PAHs: their charge distribution. A brief summary is then given.

\section{Physical processes involving PAHs}

Interstellar PAHs are abundant (they bear about $20 \%$ of the total carbon abundance in diffuse clouds: Joblin et al. 1992; Zubko et al. 2004; Draine \& Li 2007) and are the smallest grains ( 0.4 to $1 \mathrm{~nm}$ in radius) of dust grains. Because of these properties PAHs play a peculiar role in the physics and chemistry of the ISM. While discussing the key interstellar processes which involve PAHs, we will see that most of them require a detailed knowledge of the charge distribution of PAHs.

\subsection{Photoelectric heating of the gas}

Observations at $21 \mathrm{~cm}$ show that the diffuse atomic (HI) gas is at temperatures between 100 and $10^{4} \mathrm{~K}$ and in rough pressure equilibrium. To explain these temperatures, early models of the gas thermal state proposed that the HI gas was heated by cosmic rays (Field et al. 1969) but it was later realized that the flux of energetic nuclei was much ( 10 times) lower. Watson $(1972)$ then proposed that the heating of HI gas may be due to the photoelectric effect on interstellar grains.

In this process, an UV photon ejects an electron from the grain: this first step is often called photoemission. The kinetic energy carried away by the electron (typically 1 to $2 \mathrm{eV}$ ) is then rapidly deposited in the gas by inelastic collisions (in less than 10 years for proton densities $n_{H}>1 \mathrm{~cm}^{-3}$ ). To trigger photoemission, the energy of the incident photon must satisfy $h \nu>I P$ where $I P$ is the ionization potential or photoelectric threshold which depends on the charge $Z$ (algebraic number of electron charges carried by the PAH) and size $a$ of the grain. The photoelectric effect is characterized by its probability or yield and its energetic efficiency. The yield $Y_{P E}$ is the ratio of the photoemission cross-section to the absorption cross-section and depends on the energy of the photon ${ }^{1}$. Let $E_{e}$ be the mean energy of the electron ejected, the photoelectric efficiency is given by $\epsilon=Y_{P E} E_{e} / h \nu_{a}$ with $h \nu_{a}$ the mean energy of photons absorbed by the grain.

\footnotetext{
${ }^{1} \mathrm{We}$ will quote here $Y_{P E}$ for $h \nu=10 \mathrm{eV}$, roughly the energy of the maximum photoemission rate in the Mathis et al. radiation field.
} 
Interstellar grains may also capture electrons from the gas leading to an energy gas loss proportional to $k_{B} T$ per capture (see Draine 1978, Eq. (10)). This process is called grain recombination cooling. Its contribution, particularly important in warm gas, must be subtracted from the photoelectric heating rate to obtain the net photoelectric rate $\Gamma_{P E}\left(\operatorname{erg~} \mathrm{cm}^{-3} \mathrm{~s}^{-1}\right)$.

Watson (1973) further pointed out that high yields $\left(Y_{P E}>0.1\right)$ were expected for small grains because the mean free path of the electron on its way to the grain surface becomes comparable to the grain size. Early models of photoelectric heating assumed $Y_{P E} \sim 0.1$ and were successful in explaining the cold HI gas but had difficulties in supporting the warm $(T>5000 \mathrm{~K})$ gas because of the strong grain recombination cooling (Draine 1978). The photoelectric effect on PAHs does not suffer of this problem because of the higher energy of the ejected electron implying a larger cut-off temperature for the heating rate ${ }^{2}$ (Verstraete et al. 1990). Because of their small size, PAHs have a high photoelectric yield $\left(Y_{P E} \geq 0.4\right)$ and, in the neutral gas, a small mean charge both properties implying a high photoelectric efficiency (e.g., Bakes \& Tielens 1994 hereafter BT94) ${ }^{3}$. Current models of photoelectric heating show that $\epsilon$ is maximum (a few percents) in the limit of small size $(a<1 \mathrm{~nm})$ and charge $(Z<1)$ (BT94; Weingartner \& Draine 2001 hereafter WD01).

Theoretical studies of the gas thermal balance have shown that the photoelectric effect is the dominant heating process over a wide range of conditions in gas density $0.1 \leq n_{H} \leq 10^{4} \mathrm{~cm}^{-3}$ and intensity of radiation field $1 \leq G_{0} \leq 10^{6}$. On the other hand, the gas cools by collisional excitation of optically thin emission lines of major species such as $\mathrm{C}^{+}, \mathrm{O}, \mathrm{H}, \mathrm{H}_{2}$ and $\mathrm{CO}$, depending on the gas density and temperature. The photoelectric heating thus controls the gas temperature and hence the line emissivity and chemistry of interstellar clouds. Moreover, the photoelectric heating also determines the conditions under which the gas becomes thermally unstable, a key process in understanding the structure and evolution of the ISM (Audit \& Hennebelle 2005, 2010).

The hypothesis of gas photoelectric heating can be tested observationally. At densities $n_{H}>1 \mathrm{~cm}^{-3}$, the gas cools rapidly (in less than $0.1 \mathrm{Myr}$ ) and can be assumed to be in thermal equilibrium, i.e., $\Gamma_{P E}=\Lambda$ where $\Lambda$ is the gas cooling rate in erg $\mathrm{cm}^{-3} \mathrm{~s}^{-1}$. Observations of the cooling lines thus directly trace $\Gamma_{P E}$. The latter can then be compared to the dust IR emission to determine which grain sizes contribute most to the heating and with which efficiency, in order to check model predictions. Over the last decade, such studies have been performed in different contexts. Habart et al. (2001) made a detailed study of an isolated cloud with well-known properties (exciting radiation field, density profile). Using ISO-LWS observations of the major cooling lines ([CII]158 $\mu \mathrm{m}$ and $[\mathrm{OI}] 63 \mu \mathrm{m}$ ) and extracting the emission of each dust population from IRAS data, they found that the gas cooling is well correlated with the IR emission of small grains and

\footnotetext{
${ }^{2}$ The temperature at which the photoelectric heating and recombination cooling rates cancel.

${ }^{3}$ Electrostatic forces bring $I P$ close to $13.6 \mathrm{eV}$ when $Z>0$.
} 
that the photoelectric heating efficiency for PAHs was 3\% and decreases for larger grains. Modelling the [CII]158 $\mu \mathrm{m}$ emission measured by ISO-LWS towards 9 highgalactic latitude translucent clouds $\left(A_{V} \leq 5\right)$, Ingalls et al. (2002) found an overall photoelectric heating efficiency of $4.3 \%$. They also showed that the decrease of CII emission at high dust emission is due to radiative transfer inside clouds (see also Juvela et al. 2003). More recently, Rubin et al. (2009) studied the 30 Doradus star forming region in the Large Magellanic Cloud with Spitzer IRAC+MIPS (dust emission) and BICE data (CII emission). They also found that PAHs have the highest photoelectric efficiency, about 5\%. Finally, in external normal galaxies, a correlation has been found between the CII line and the 5-to-10 $\mu \mathrm{m}$ ISOCAM emission ascribed to PAHs (Helou et al. 2001). All these results are in good agreement with model predictions of the photoelectric effect on dust grains and show the dominant role of PAHs for the gas heating.

\subsection{Screening of UV radiation}

Aromatic species like PAHs show strong UV absorption in two broad bands centered at $200 \mathrm{~nm}\left(\pi^{*} \leftarrow \pi\right.$ transitions) and $70 \mathrm{~nm}\left(\sigma^{*} \leftarrow \sigma\right.$ transitions $)$. PAHs are thus good candidates to explain the $217 \mathrm{~nm}$ bump and the far-UV rise of the interstellar extinction curve as suggested by recent theoretical and laboratory studies (see the review by Mulas et al. this volume and Cecchi-Pestellini et al. 2008; Steglich et al. 2009). In particular, PAHs absorb efficiently dissociating photons (at $\lambda \leq 100 \mathrm{~nm}$ ) thus controlling the molecular fraction and excitation of interstellar clouds. This UV screen will be enhanced if PAHs are more abundant at cloud boundaries as suggested by observations (the limb brightening effect, see Sect. 1). As discussed by Mulas et al. (this volume), the far-UV absorption of PAHs depends on their charge state.

\subsection{Ionization of dense gas}

The delocalized $\pi$-electrons of PAHs are easily polarizable (Omont 1986). Thus due to their high polarizability and abundance PAHs can efficiently capture electrons and, in shielded interstellar regions, PAH anions may be as abundant as electrons $\left(x=n_{e} / n_{H} \sim 10^{-7}\right)$. Charge exchange between positive ions generated by cosmic rays in the gas and $\mathrm{PAH}$ anions quenches the ion-molecule chemistry and may reconcile observations and models (Bakes \& Tielens 1998; Flower et al. 2007; Wakelam \& Herbst 2009; Goicoechea et al. 2009). The role of PAHs in interstellar chemistry is discussed by Bierbaum et al. (this volume). PAH anions may also induce recombination of CII, hence leading to warmer gas in diffuse clouds (Wolfire et al. 2008).

Charged PAHs are also important for the ion-neutral coupling and the dynamics. In magnetic C-shocks, PAHs may produce a shortage of electrons leading to the decoupling of larger grains in the shock tail. At a given density, this will increase the Alfven velocity in the shock and its emissivity by the ion-neutral drift (Ciolek et al. 2002, 2004; Guillet et al. 2007). In the context of protostars, the 
role of PAHs in braking the collapse has been studied by Ciolek and collaborators. At cloud boundaries where UV photons are less shielded, PAHs may carry positive as well as negative charges (see Sect. 3): this could accelerate the coagulation of PAHs together as suggested by the observations of Flagey et al. (2009).

\section{$2.4 \mathrm{H}_{2}$ formation}

It has long been known that, at ISM densities, $\mathrm{H}_{2}$ cannot form efficiently in the gas phase. In 1963, Gould \& Salpeter proposed that $\mathrm{H}_{2}$ may form by catalytic reaction on interstellar grains where an $\mathrm{H}$ atom bound to the grain surface recombines with another H. Molecular hydrogen has a profound impact on the physics and chemistry of the ISM: it efficiently cools the gas (Le Bourlot et al. 1999) and acts as a shield to photodissociating UV photons, initiating the formation of $\mathrm{CO}$ and of chemistry. In spite of many theoretical and experimental studies, the detailed mechanism by which $\mathrm{H}_{2}$ forms is complex and still debated because it depends on the structure and composition of the grains (surface roughness, presence of chemior physisorption sites for $\mathrm{H}$ ) and involves the issue of distributing the $4.5 \mathrm{eV}$ bonding energy of the molecule between excitation energy (grain, $\mathrm{H}_{2}$ vibrational or rotational) and $\mathrm{H}_{2}$ kinetic energy (see Bierbaum et al., Thrower et al. this volume). The rate coefficient for $\mathrm{H}_{2}$ formation is observed to vary from a few tenths to a few times the standard value of Jura (1975), $R_{0}=3 \times 10^{-17} \mathrm{~cm}^{3} \mathrm{~s}^{-1}$ (Gry et al. 2002; Browning et al., Habart et al. 2003; Gillmon et al. 2006; Thi et al. 2009). In addition, the observations of $\mathrm{H}_{2}$ emission in excited regions favor formation routes involving chemisorbed sites for $\mathrm{H}$ (Habart et al. 2004).

PAHs are attractive candidates for the formation of $\mathrm{H}_{2}$ because they represent two thirds of the total grain surface, e.g., Habart et al. 2004) and carry chemically bonded $\mathrm{H}$ atoms. Observations indicate an expected trend between $\mathrm{H}_{2}$ and PAH integrated emissions (Habart et al. 2004) but the corresponding emissivity profiles are not always correlated, (Habart et al. 2003; Berné et al. 2009; Velusamy \& Langer 2008). These results may be related to the radiative transfer of UV photons across cloud borders with different density profiles. Shock excited $\mathrm{H}_{2}$ emission does not suffer of this limitation and should represent a better test (Guillard et al. 2010). Analysing UV absorption data in diffuse clouds, Wolfire et al. (2008) modelled in a consistent way the $\mathrm{C} / \mathrm{C}^{+}$and $\mathrm{HI} / \mathrm{H}_{2}$ transitions with $\mathrm{PAH}$ assisted recombination of $\mathrm{C}^{+}$and photoelectric heating. Their results also indicate a correlation between the $\mathrm{PAH}$ abundance and the $\mathrm{H}_{2}$ formation rate.

\subsection{Spinning dust emission and microwave polarization}

At centimetric wavelengths (around $30 \mathrm{GHz}$ ), an emission component discovered by Kogut et al. (1996) in COBE data was later shown to correlate with the emission of large dust grains (Leitch et al.; de Oliveira-Costa et al. 1997). Unexpected in this spectral range where the free-free, synchrotron and large grain emissions usually dominate, this component was dubbed anomalous emission. Due to this mixing, the existence of anomalous emission has long been debated and it is only 
recently that it has been extracted in all-sky WMAP data thanks to the constraints on polarization (Miville-Deschênes et al. 2008). Understanding this emission is particularly important because it appears in a spectral region where fluctuations of the cosmic microwave background are best measured. It is now further traced by the measurements of the Planck mission.

Several emission mechanisms have been proposed to explain the anomalous emission. Draine \& Lazarian (1998a) considered the rotational emission of PAHs (or spinning dust emission). Draine \& Lazarian (1999) further proposed magnetic dipole emission of grains with magnetic inclusions and Jones (2009) proposed low-energy transitions arising in disordered grains. The latter two mechanisms involve large grains $(a \sim 0.1 \mu \mathrm{m})$. Recent analysis of observations indicate that the anomalous emission correlates better with the mid-IR emission due to PAHs (Casassus et al. 2006, 2009; Ysard et al. 2010) than with the emission of larger grains. Another hint comes from polarization: the anomalous emission appears to be weakly polarized (Battistelli et al. 2006) as expected for small grains (Lazarian \& Draine 2000; Martin et al. 2007) thus favoring the spinning dust explanation ${ }^{4}$. These results have stimulated new theoretical studies of PAH rotational dynamics in the ISM (Ali-Haïmoud et al. 2009; Ysard \& Verstraete; Hoang et al.; Silsbee et al. 2010) which confirmed the main features of the Draine \& Lazarian (1998b) model. Namely, the PAH rotation is mostly controlled by gas-grain collisions and, in irradiated regions, also by their vibrational (IR) emission. In addition, these processes depend on the PAH charge.

The anomalous emission may thus trace the PAH abundance and provide new constraints on PAHs such as their permanent electric dipole moment. Further on, the contribution of spinning PAHs could reduce the polarization fraction measured in the microwave by Planck. Quantitative modelling of this emission component is necessary for the analysis of the Planck polarization data in the cosmological context but also for ISM studies (magnetic field).

\section{The charge distribution of PAHs}

Most of the processes discussed in the previous section require a detailed knowledge of the PAH charge distribution. This is also true for the spectroscopic properties of PAHs, from the IR to the UV (Pauzat, Pino et al., Mulas et al., Oomens this volume). We therefore review the current state-of-the-art in modelling the charge of interstellar PAHs.

In the ISM PAHs become charged because of photoemission or collisions with charged species. Excluding dark clouds (where PAHs may have coagulated on larger grains), the latter case involves the most abundant species $e^{-}, \mathrm{H}^{+}$and $\mathrm{C}^{+}$. Since the characteristic times for charge fluctuations are short, a stationary description is often adopted (see Ivlev et al. 2010 however for a discussion of the

\footnotetext{
${ }^{4}$ Conversely, large grains are known to polarize light in emission (FIR to submm) and extinction (IR to UV), indicating that grains are not spherical and aligned with the magnetic field.
} 

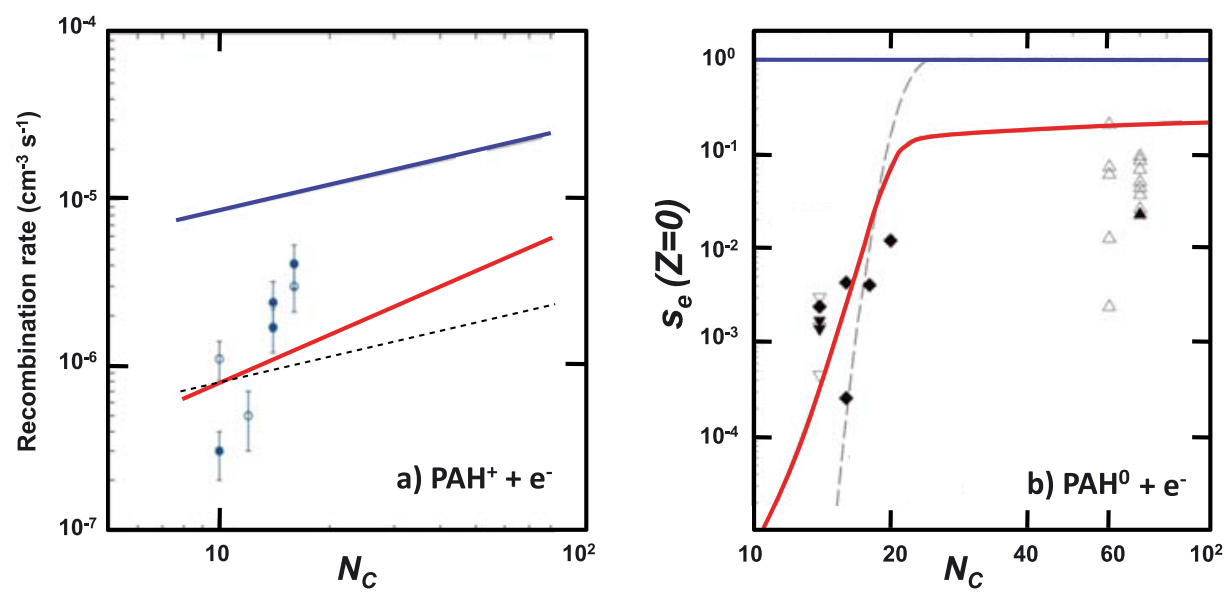

Fig. 1. a) Rate coefficient at $300 \mathrm{~K}$ as a function of size (in number of $\mathrm{C}$ atoms per molecule) for the electronic recombination of $\mathrm{PAH}^{+}$(adapted from Tielens 2008). The data points are from Biennier et al. (2006). The bottom solid line shows the law adopted by WD01 and the top solid line that of BT94 $\left(s_{e}=1\right)$. The dashed line shows the $s_{e}=0.1$-case. b) Sticking coefficient for electronic capture on a neutral PAH as a function of size (adapted from WD01, note that triangles at large sizes are for $\mathrm{C}_{60}$ and $\mathrm{C}_{70}$ ). Solid lines are as in a). The dashed line shows the law of Dartois \& d'Hendecourt (1997).

effect of charge fluctuations). The charge distribution $f(Z)$ is then derived from the balance equation (BT94):

$$
f(Z)\left[J_{P E}(Z)+J_{i}(Z)\right]=f(Z+1) J_{e}(Z+1)
$$

written for a given PAH size and where the $J$-terms represent charge currents. Estimates of the latter require the knowledge of rate coefficients which depends on intrinsic quantities such as the photoelectric yield, the threshold $I P$ and the electron capture rate coefficient (or sticking coefficient) (see Sect. 2.1). Two types of models have been developed: (i) molecular models using the properties measured in the laboratory on small, real molecules (Allain et al., Salama et al. 1996; Le Page et al. 2001, 2003) and, (ii) astrophysical models treating PAHs as generic species whose properties follow size trends based on laboratory measurements (BT94; Dartois \& d'Hendecourt 1997; WD01; van Hoof et al. 2004). Being more widely applied, we focus here on the models of BT94 and WD01.

The photoelectric (or photoionization) yield for PAHs has been measured on a few, small (containing less than 25C atoms) PAHs (Verstraete et al. 1990; Jochims et al. 1996). The expressions used by BT94 and WD01 provide a good description of these data and extrapolate well to measurements on larger $(a \geq 100 \mathrm{~nm})$ grains (Abbas et al. 2006). The functions for the photoelectric threshold $I P(Z, a)$ used in both models are also similar with the exception of the electron affinity $(Z=-1)$, 

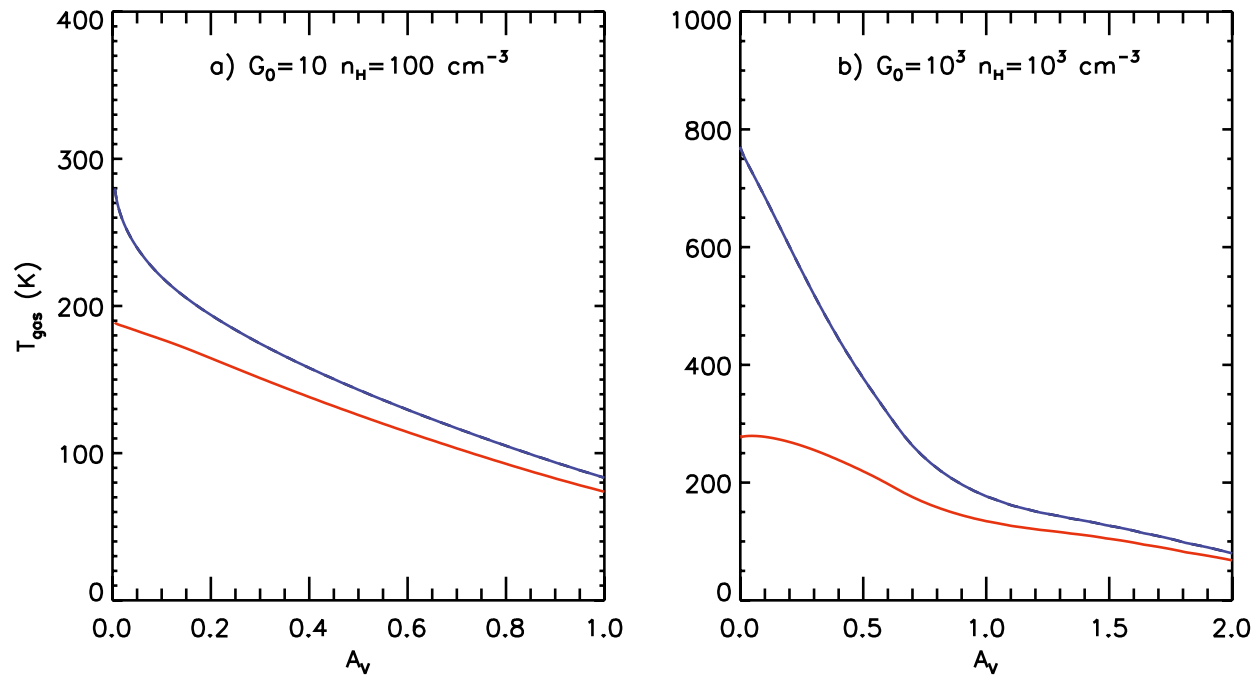

Fig. 2. Temperature profile as a function of optical depth for two PDRs of a) low and b) high excitation. In both panels, the bottom (top) solid line shows the WD01 (BT94) case respectively.

somewhat overestimated by BT94. It is worth noting that these thresholds do not apply to fullerenes such as $\mathrm{C}_{60}, \mathrm{C}_{70}$ (BT94).

The situation is different for the sticking coefficient (or probability) $s_{e}$ of the electronic capture: WD01 use a size dependent coefficient whereas BT94 assume $s_{e}=1$ for all sizes. These two prescriptions are compared to laboratory data in Figure 1 as a function of molecular size. Note that for interstellar PAHs, one has $N_{C}>15$ (Le Page et al. 2003). The prescription of BT94 leads to a more efficient electronic capture and hence more abundant neutral or anionic species. We note that the laboratory measurements for $\mathrm{PAH}^{+}$recombination fall between the BT94 and WD01 prescriptions which can therefore be seen as extreme cases. Another consequence is that the photoelectric heating rate of BT94 is stronger than that of WD01 because PAHs are less positively charged in the former case (see Sect. 2.1). We illustrate this in Figure 2 by comparing the gas temperature across two photodissociated interfaces or photon-dominated regions (PDR) computed with the Meudon PDR code (Le Petit et al. 2006). The physics and chemistry of such regions depend on the gas density $n_{H}$ and on the intensity of the UV radiation field represented by $G_{0}{ }^{5}$. We ran isochoric models irradiated on a single side (see Fig. 2) and with the following gas abundances (in ppm): $[\mathrm{C}]=130,[\mathrm{O}]=320$, $[\mathrm{N}]=75,[\mathrm{Mg}]=35,[\mathrm{Fe}]=28,[\mathrm{Si}]=1$. As expected, in the BT94 case the mean charge

\footnotetext{
${ }^{5} G_{0}$ is a scaling factor for the energy density of the radiation field integrated between 6 and $13.6 \mathrm{eV} ; G_{0}=1$ corresponds to the standard radiation field of Mathis et al. (1983).
} 

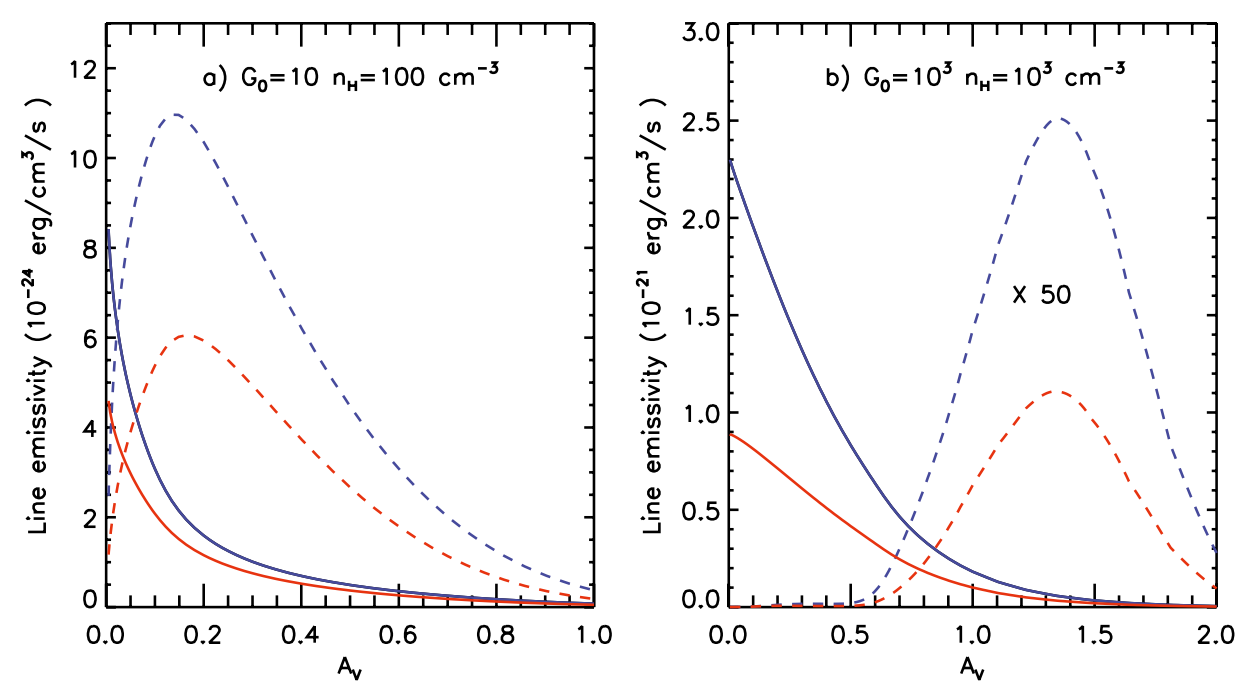

Fig. 3. Line emissivity profiles as a function of optical depth in two PDRs. As in Fig. 2, the bottom (top) solid and dashed lines represent the case of WD01 (BT94). The solid line shows the [OI]63 $\mu \mathrm{m}$ emissivity and the dashed line shows the emissivity in the first 4 rotational lines of $\mathrm{H}_{2}$. For clarity the $\mathrm{H}_{2}$ emissivities have been multiplied by 50 .

$<Z>$ of a $20 \mathrm{C}$ PAH is shifted by -0.5 to -1 with respect to the WD01 case ${ }^{6}$. The photoelectric heating rate of BT94 is correspondingly higher (2 to 4 times higher than WD01 for the present $G_{0}=10$ and $10^{3}$ PDRs respectively). Even for conditions of low excitation $\left(G_{0}=10\right)$, the BT94 model produces significant column densities of warm gas. In an edge-on geometry, the line emissivity (in erg $\mathrm{cm}^{-3} \mathrm{~s}^{-1}$ ) of major gas coolants is significantly affected as illustrated in Figure 3. Integrated line fluxes (face-on geometry) are less affected (below $30 \%$ variations). Such differences will lead to quite different diagnostics of the physical conditions (intensity of the UV radiation field, gas density and temperature) and emphasize the need for more laboratory measurements of the electronic capture on astrophysically relevant PAHs (containing more than 20C). Such measurements should also be carried at different temperatures to check that the classical limit often used provides a reasonable description ${ }^{7}$.

Thanks to the contrasted behaviour of the CC-to-CH ratio of the vibrational band intensities in neutral and ionized PAHs (e.g., Bauschlicher et al. 2008 and Pauzat this volume), the charge distribution of PAHs can be constrained from mid-IR observations. Flagey et al. (2006) found that, in the diffuse ISM, 40\% of PAHs are ionized. Analyzing a sample of galactic and extragalactic excited

\footnotetext{
${ }^{6}$ With the WD01 model, the mean charge is $+0.5(+0.9)$ for the $G_{0}=10\left(10^{3}\right)$ PDR respectively.

${ }^{7}$ Some data suggest a behaviour departing from the classical $T^{1 / 2}$ (Biennier et al. 2006).
} 
regions, Galliano et al. (2008 and Galliano this volume) found the expected trend between the fraction of ionized PAHs and excitation conditions.

\section{Summary}

PAHs have a strong impact on the ISM: they dominate the gas heating in irradiated regions and carry a significant fraction of the dust surface, a potential site for the formation of $\mathrm{H}_{2}$ and other molecules. In dense clouds, PAHs may become an important sink for electrons and control the ion-molecule chemistry as well as the dynamics of neutrals relative to ionized species in presence of a magnetic field. PAHs are therefore key to understanding the evolution of interstellar gas. Most of the processes involving PAHs depend on their charge state and this is also true of their spectroscopical properties. Quantitative state-of-the-art models of the PAH charge are limited by the lack of data on the rate coefficient for the electronic capture on large species containing more than 20 carbon atoms (see also Montillaud et al. this volume). This leads to significant uncertainties in deriving the physical conditions and line emission of interstellar clouds. More laboratory measurements are needed in this area.

The author is grateful to Vincent Guillet and Jacques Le Bourlot for useful discussions and help while preparing this review.

\section{References}

Abbas, M.M., et al., 2006, ApJ, 645, 324

Ali-Haïmoud, Y., Hirata, C.M., \& Dickinson, C., 2009, MNRAS, 395, 1055

Allain, T., Leach, S., \& Sedlmayr, E., 1996, A\&A, 305, 616

Audit, E., \& Hennebelle, P., 2005, A\&A, 433, 1

Audit, E., \& Hennebelle, P., 2010, A\&A, 511, A76

Bakes, E.L.O., \& Tielens, A.G.G.M., 1994, ApJ, 427, 822 (BT94)

Bakes, E.L.O., \& Tielens, A.G.G.M., 1998, ApJ, 499, 258

Bauschlicher, C.W., Jr., Peeters, E., \& Allamandola, L.J., 2008, ApJ, 678, 316

Battistelli, E.S., Rebolo, R., Rubiño-Martín, J.A., et al., 2006, ApJ, 645, L141

Bernard, J.P., Boulanger, F., \& Puget, J.L., 1993, A\&A, 277, 609

Berné, O., et al., 2007, A\&A, 469, 575

Berné, O., Fuente, A., Goicoechea, J.R., et al., 2009, ApJ, 706, L160

Biennier, L., Alsayed-Ali, M., Foutel-Richard, A., et al., 2006, Chem. Evolu. Universe, Faraday Discuss., 133, 289

Boulanger, F., Falgarone, E., Puget, J.L., \& Helou, G., 1990, ApJ, 364, 136

Boulanger, F., Prevot, M.L., \& Gry, C., 1994, A\&A, 284, 956

Browning, M.K., Tumlinson, J., \& Shull, J.M., 2003, ApJ, 582, 810

Casassus, S., Cabrera, G.F., Förster, F., et al., 2006, ApJ, 639, 951

Casassus, S., et al., 2008, MNRAS, 391, 1075 
Ciolek, G.E., \& Roberge, W.G., 2002, ApJ, 567, 947

Ciolek, G.E., Roberge, W.G., \& Mouschovias, T.C., 2004, ApJ, 610, 781

Compiègne, M., Abergel, A., Verstraete, L., \& Habart, E., 2008, A\&A, 491, 797

Dartois, E., \& D'Hendecourt, L., 1997, A\&A, 323, 534

de Oliveira-Costa, A., Kogut, A., Devlin, M.J., et al., 1997, ApJ, 482, L17

Draine, B.T., 1978, ApJS, 36, 595

Draine, B.T., \& Lazarian, A., 1998a, ApJ, 494, L19

Draine, B.T., \& Lazarian, A., 1998b, ApJ, 508, 157

Draine, B.T., \& Li, A., 2007, ApJ, 657, 810

Field, G.B., Goldsmith, D.W., \& Habing, H.J., 1969, ApJ, 155, L149

Flagey, N., Boulanger, F., Verstraete, L., et al., 2006, A\&A, 453, 969

Flagey, N., et al., 2009, ApJ, 701, 1450

Flower, D.R., Pineau Des Forêts, G., \& Walmsley, C.M., 2007, A\&A, 474, 923

Geers, V.C., et al., 2006, A\&A, 459, 545

Geers, V.C., van Dishoeck, E.F., Pontoppidan, K.M., et al., 2009, A\&A, 495, 837

Gillmon, K., Shull, J.M., Tumlinson, J., \& Danforth, C., 2006, ApJ, 636, 891

Goicoechea, J.R., Pety, J., Gerin, M., Hily-Blant, P., \& Le Bourlot, J., 2009, A\&A, 498, 771

Gould, R.J., \& Salpeter, E.E., 1963, ApJ, 138, 393

Gry, C., Boulanger, F., Nehmé, C., et al., 2002, A\&A, 391, 675

Guillard, P., Boulanger, F., Cluver, M.E., et al., 2010 [arXiv:1004.0677]

Guillet, V., Pineau Des Forêts, G., \& Jones, A.P., 2007, A\&A, 476, 263

Habart, E., Verstraete, L., Boulanger, F., et al., 2001, A\&A, 373, 702

Habart, E., Boulanger, F., Verstraete, L., et al., 2003, A\&A, 397, 623

Habart, E., Boulanger, F., Verstraete, L., Walmsley, C.M., \& Pineau des Forêts, G., 2004, A\&A, 414, 531

Hoang, T., Draine, B.T., \& Lazarian, A., 2010, ApJ, 715, 1462

Ingalls, J.G., Reach, W.T., \& Bania, T.M., 2002, ApJ, 579, 289

Ivlev, A.V., Lazarian, A., Tsytovich, V.N., et al., 2010 [arXiv: 1003.2633]

Jochims, H.W., Baumgaertel, H., \& Leach, S., 1996, A\&A, 314, 1003

Jones, A.P., 2009, A\&A, 506, 797

Jura, M., 1975, ApJ, 197, 575

Juvela, M., Padoan, P., \& Jimenez, R., 2003, ApJ, 591, 258

Kogut, A., Banday, A.J., Bennett, C.L., et al., 1996, ApJ, 460, 1

Lazarian, A., \& Draine, B.T., 2000, ApJ, 536, L15

Le Bourlot, J., Pineau des Forêts, G., \& Flower, D.R., 1999, MNRAS, 305, 802

Lebouteiller, V., Brandl, B., Bernard-Salas, J., Devost, D., \& Houck, J.R., 2007, ApJ, 665,390

Leitch, E.M., Readhead, A.C.S., Pearson, T.J., \& Myers, S.T., 1997, ApJ, 486, L23

Le Page, V., Snow, T.P., \& Bierbaum, V.M., 2001, ApJS, 132, 233

Le Page, V., Snow, T.P., \& Bierbaum, V.M., 2003, ApJ, 584, 316

Le Petit, F., Nehmé, C., Le Bourlot, J., \& Roueff, E., 2006, ApJS, 164, 506 
Martin, P.G., 2007, EAS Publications Series, 23, 165

Mathis, J.S., Mezger, P.G., \& Panagia, N., 1983, A\&A, 128, 212

Miville-Deschênes, M.-A., Ysard, N., Lavabre, A., et al., 2008, A\&A, 490, 1093

Omont, A., 1986, A\&A, 164, 159

Peeters, E., Martín-Hernández, N.L., Rodríguez-Fernández, N.J., \& Tielens, X., 2005, Sp. Science Rev., 119, 273

Rapacioli, M., Joblin, C., \& Boissel, P., 2005, A\&A, 429, 193

Rubin, D., et al., 2009, A\&A, 494, 647

Salama, F., Bakes, E.L.O., Allamandola, L.J., \& Tielens, A.G.G.M., 1996, ApJ, 458, 621

Silsbee, K., Ali-Haimoud, Y., \& Hirata, C.M., 2010 [arXiv: 1003.4732]

Tielens, A.G.G.M., 2008, ARA\&A, 46, 289

Thi, W.-F., van Dishoeck, E.F., Bell, T., Viti, S., \& Black, J., 2009, MNRAS, 400, 622

van Hoof, P.A.M., Weingartner, J.C., Martin, P.G., Volk, K., \& Ferland, G.J., 2004, MNRAS, 350, 1330

Velusamy, T., \& Langer, W.D., 2008, AJ., 136, 602

Verstraete, L., Léger, A., d'Hendecourt, L., Défourneau, D., \& Dutuit, O., 1990, A\&A, 237,436

Wakelam, V., \& Herbst, E., 2008, ApJ, 680, 371

Watson, W.D., 1972, ApJ, 176, 103

Watson, W.D., 1973, J. Opt. Soc. Amer. (1917-1983), 63, 164

Weingartner, J.C., \& Draine, B.T., 2001, ApJS, 134, 263 (WD01)

Wolfire, M.G., Tielens, A.G.G.M., Hollenbach, D., \& Kaufman, M.J., 2008, ApJ, 680, 384

Ysard, N., Miville-Deschênes, M.A., \& Verstraete, L., 2010, A\&A, 509, L1

Ysard, N., \& Verstraete, L., 2010, A\&A, 509, A12

Zubko, V., Dwek, E., \& Arendt, R.G., 2004, ApJS, 152, 211 